\title{
Type Ib/c supernovae observations with VLBI
}

\author{
Z. Paragi ${ }^{* \dagger}$ \\ ${ }^{1}$ JIVE, Dwingeloo, Netherlands \\ ${ }^{2}$ MTA Research Group for Physical Geodesy and Geodynamics, Penc, Hungary \\ E-mail: zparagi@jive.nl
}

\section{Alexander J. van der Horst}

NASA Postdoctoral Program Fellow, NASA/MSFC/ORAU, Huntsville, AL, USA

E-mail: alexander.j.vanderhorst@nasa.gov

\section{Chryssa Kouveliotou}

NASA/MSFC, Huntsville, AL, USA

E-mail: chryssa.kouveliotou@nasa.gov

\section{Jonathan Granot}

University of Hertfordshire, UK

E-mail: j.granot@herts.ac.uk

\section{Enrico Ramirez-Ruiz}

University of California, Santa Cruz, USA

E-mail: enrico@ucolick.org

\section{Greg Taylor}

University of New Mexico, Albuquerque, USA

E-mail: gbtaylor@unm.edu

\begin{abstract}
Supernovae ( $\mathrm{SNe}$ ) Ib/c have drawn increasing attention since 1998 owing to their sparse association with long duration gamma-ray bursts (GRBs). Although both phenomena originate from the core collapse of a massive star, the former release most of their energy at optical wavelengths, while the latter do it mostly in soft gamma-rays or hard X-rays. Moreover, the GRB central engine generates ultra-relativistic jets, which beam the early emission into a small narrow cone; no relativistic outflows have yet been found in $\mathrm{SNe} \mathrm{Ib} / \mathrm{c}$ explosions. We show results from very long baseline interferometry (VLBI) observations of three supernovae, one of which appears to have produced a collimated, mildly relativistic outflow.
\end{abstract}

ISKAF2010 Science Meeting - ISKAF2010

June 10-14, 2010

Assen, the Netherlands

\footnotetext{
*Speaker.

${ }^{\dagger}$ We report these results for a greater collaboration.
} 


\section{Introduction}

Gamma-ray bursts of the long duration class (LGRBs) and the less energetic X-ray flashes (XRF) are thought to be caused by a relativistic jet emerging from the collapse of a massive star (e.g. the collapsar model, Woosley 1993). While the initial burst of high energy emission is due to internal shocks, afterglow emission from X-rays to the radio originates in shocks as the blastwave interacts with the ambient medium (Meszaros \& Rees 1997; Wijers et al. 1997). Until recently there have been four firm associations between LGRBs/XRFs and type $\mathrm{Ib} / \mathrm{c}$ supernovae: GRB 970508/SN 1998bw (Galama et al. 1998), GRB 030329/SN 2003dh (Hjorth et al 2003; Stanek et al. 2003), GRB 031203/SN 20031w (Malesani et al. 2004), XRF 060218/SN and 2006aj (Pian et al. 2006). The case of XRF 080109/SN 2008D we will discuss below, and there are two new associations found in 2010: XRF 100316D/SN 2010bh (Starling et al. 2010; Fan et al. 2010), and possibly GRB 091127/SN 2009nz (Cobb et al. 2010). A detailed study of the first four associations revealed that while the total energy in these supernovae are very similar, only a very small fraction of that energy may end up in highly relativistic ejecta, that explains the relatively low GRB event rate in SNe (Kaneko et al. 2007). Another possibility is that some of the SNe are producing relativistic ejecta at large angles to the line of sight. As a result, the emission would be initially strongly beamed away from our line of sight and only the late time radio emission could be detected (Pacziński 2001; Granot \& Loeb 2003).

Since type $\mathrm{Ib} / \mathrm{c} \mathrm{SNe}$ are usually faint (observed at most at $\sim$ mJy levels) in the radio, evolve quickly, and are typically located at large distances from us, direct measurement of their expansion by interferometry imaging has been very challenging. The SKA may thus make a very significant contribution in this area in the future, with its superior sensitivity and its large field of view capability to identify these sources in radio surveys of nearby galaxies, and monitor them frequently before they fade away. Here we report on very long baseline interferometry (VLBI) imaging results with the European VLBI Network (EVN) and other telescopes (see below) of two type Ib/c SNe that offered a unique opportunity to probe their expansion because they were amongst the closest ever $\mathrm{SNe} \mathrm{Ib} / \mathrm{c}$ observed so far.

\section{Size constraints on XRF 080109/SN 2008D}

On 9 January 2008 a new X-ray source was discovered by Swift during the observations of SN 2007uy in NGC 2770 (Berger \& Soderberg 2008). The transient was initially thought to belong in the class of X-ray flash sources (thus the designation XRF 080109), a sub-class of the enigmatic gamma-ray bursts (GRBs). As the optical counterpart brightened, spectroscopic observations became possible and it was classified as a type Ic supernova (Malesani et al. 2008; Valenti et al. 2008a) and designated SN 2008D. With later appearance of Helium lines it was re-classified to type Ib (e.g. Mazzali et al. 2008). The source was extensively observed in the radio regime following its detection with the VLA (Soderberg 2008) and the WSRT (van der Horst et al. 2008), along with SN2007uy. NGC 2770 lies at a redshift of 0.007 or a distance of about $27 \mathrm{Mpc}$. The relative closeness of the system made these objects ideal targets for VLBI imaging. 

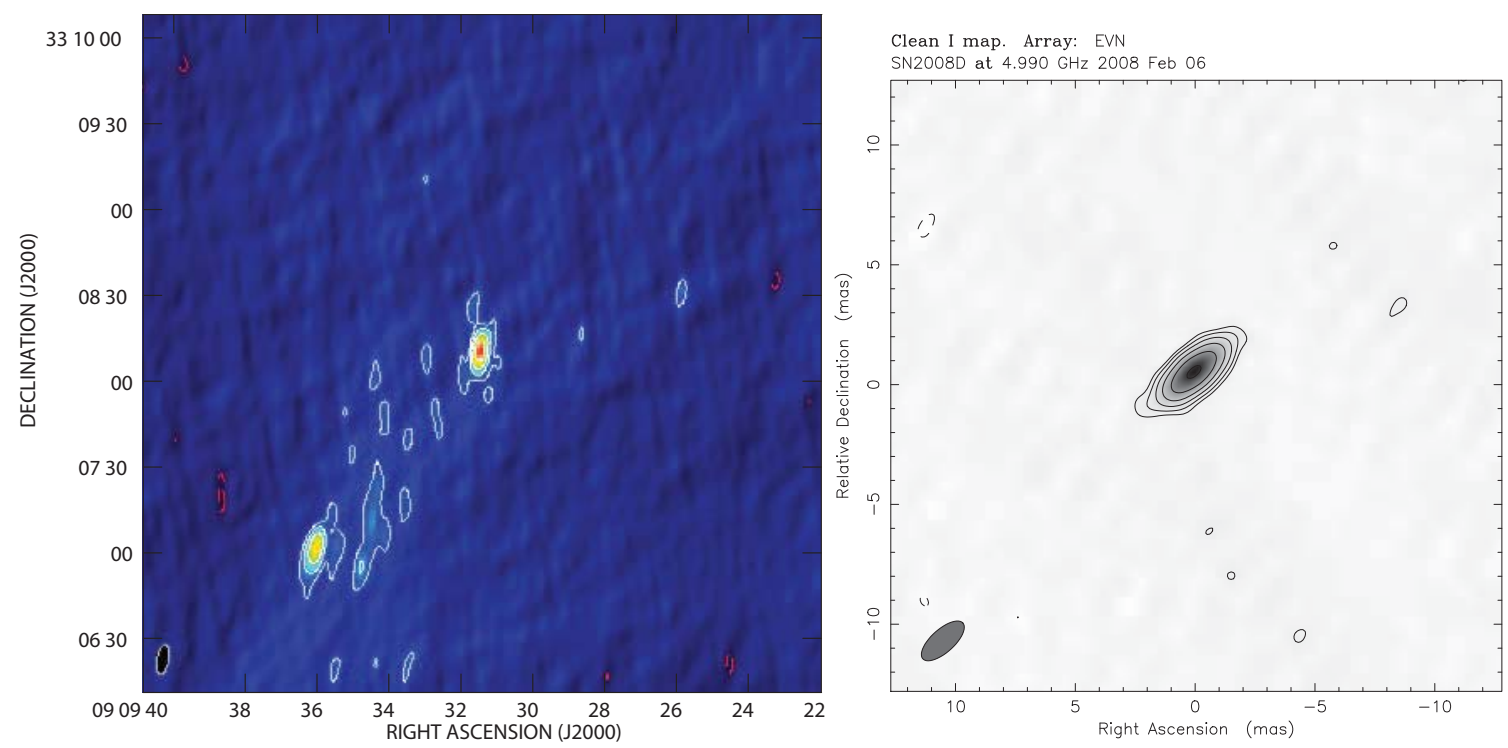

Figure 1: Left: Westerbork synthesis array map of XRF 080109/SN 2008D (centre of image) and SN 2007uy (lower left on image) in the nearby galaxy NGC 2770. The beam was $10.6 \times 4.2$ arcseconds, PA=-10.3 degree. The data were recorded parallel to the VLBI observations. The main target source was about 2.1 mJy on 6 February 2008, and decreased to 0.9 mJy by 18 March 2008. Right: EVN plus Hancock and St. Croix from the VLBA image of XRF 080109/SN 2008D on 6 February 2008. The beam was $2.24 \times 0.96$ milliarcseconds, $\mathrm{PA}=-48.2$ degree. Model-fitting indicated a marginally resolved source, but it was still consistent with a point source within the errors.

\subsection{VLBI observations}

We carried out observations at $5 \mathrm{GHz}$ on 6 February and 18 March 2008, 28 and 69 days after the discovery of XRF 080109/SN 2008D, respectively. The participating telescopes were Arecibo, Effelsberg, Jodrell Bank (MkII), Hartebeesthoek, Medicina, Noto, Onsala, Torun and Westerbork from the EVN, Hancock and St. Croix from the VLBA. The aggregate bitrate was $1024 \mathrm{Mbps}$ using 2-bit sampling at the EVN stations, the VLBA telescopes recorded at a rate of $512 \mathrm{Mbps}$ with 1-bit sampling. Total flux density measurements were possible using the synthesis array data of the WSRT. The targets were phase-referenced to the nearby calibrators J0911+3349 (0.8 deg.) and J0919+3324 (2 deg.). The main target SN 2008D was observed for $\sim 200$ minutes total and SN 2007uy (as a check source) for a shorter time of about 40 minutes. The phase-referencing cycle was 3:30-1:30 minutes for the targets and the more nearby calibrator, including a 1 minute scan on the second calibrator as well in every other cycle. We followed the standard data reduction methods in NRAO AIPS, model-fitting was carried out in Difmap (Shepherd et al. 1994).

\subsection{Size contraints from model-fitting and Monte-Carlo simulatons}

The VLBI images at the two epochs show unresolved sources (WSRT and VLBI maps are shown for the first epoch in Figure 1). Phase-referencing worked well, in particular, the observed VLBI peak brightnesses of SN 2008D of $2.0 \mathrm{mJy} / \mathrm{beam}$ and $0.9 \mathrm{mJy} / \mathrm{beam}$ (off source noise $\sim 25 \mu \mathrm{Jy} / \mathrm{beam}$ ) at the two epochs agreed very well with the WSRT total flux density measurements 
of $2.09 \pm 0.06 \mathrm{mJy}$ and $0.86 \pm 0.06 \mathrm{mJy}$, respectively. Measuring the source sizes in model-fitting was less straightforward because various phase-referencing strategies provided different results, ranging from practically point source to a few tenth of milliarcseconds. We carried out MonteCarlo simulations which showed that assuming thermal noise only SN 2008D might have been marginally resolved. However since the effect of likely phase errors in the phase-referencing process have not been included, we take the measured sizes as upper limits. The upper limits of 0.4 mas at 28 days and 0.5 mas at 69 days in SN 2008D translate to an overal upper limit of $0.57 \mathrm{c}$ to the average isotropic expansion speed. From the upper limit on proper motion we derive an expansion upper limit of $0.38 \mathrm{c}$ between the two epochs. These results are consistent with the analysis of radio lightcurve as well as the VLBA and HSA measurements (Soderberg et al. 2008, Bietenholz et al. 2009). For SN 2007uy only a reliable angular size upper limit of 0.3 mas could be obtained at 37 days, which translates to an upper limit in average expansion speed of 0.64c. Our observations show that these two supernovae were at most mildly relativistic (van der Horst et al., submitted to ApJ).

\section{The curious case of SN $2007 \mathrm{gr}$}

Our next source, SN 2007gr was discovered by the Katzman Automatic Imaging Telescope (KAIT) on 15 August 2007 (Madison \& Li 2007). It was found in the bright spiral galaxy NGC 1058 , which is located only $10.6 \pm 1.3 \mathrm{Mpc}$ away from us (Pilyugin et al. 2004), i.e. almost three times closer than the two sources in NGC 2770 described above. Based on the non-detection of He lines in the spectrum, SN 2007gr was classified as a type Ic stripped envelope core-collapse supernova (Crockett et al. 2008, Valenti et al. 2008b), and as such it was one of the closest of its kind. Following the early optical discovery (the source was less than 5 days old), it was soon detected by the Very Large Array (VLA) on 17 August 2007 at $4.8 \mathrm{GHz}$ with a flux density of $610 \pm 40 \mu \mathrm{Jy}$ (Soderberg 2007), still in the rising phase of the radio lightcurve.

\subsection{The initial e-VLBI and the follow-up EVN+GBT measurements}

Arranging VLBI observations with the most sensitive radio telescopes on a short notice is not always straightforward. We made use of the (at that time) developing electronic-VLBI (e-VLBI) mode (e.g. Szomoru 2008) offered by the EVN to observe the source on 6-7 September 2007 at $5 \mathrm{GHz}$. Historically, these were the first sensitive continuum astronomical observations with eVLBI that achieved a data rate of $256 \mathrm{Mbps}$. The array consisted of Darnhall, Jodrell Bank (MkII), Medicina, Onsala (25m), Torun and the WSRT ${ }^{1}$. The source was phase-referenced to the nearby calibrator J0253+3835 in 1.5-5 minute cycles. As was reported in Paragi et al. 2010 (hereafter paper I), SN $2007 \mathrm{gr}$ was detected with a peak brightness of $422 \mu \mathrm{Jy} /$ beam beam at 5.6 times the offsource noise level of $75 \mu \mathrm{Jy} / \mathrm{beam}$, and it appeared compact. The upper limit of 7 milliarcseconds (mas) for its angular diameter size corresponds to a linear size of $<1.1 \times 10^{18} \mathrm{~cm}$ at $10.6 \mathrm{Mpc}$ about 25 days after the explosion, which sets an upper limit of $<8.6 \mathrm{c}$ to the average isotropic apparent expansion speed of the ejecta.

\footnotetext{
${ }^{1}$ At present most of the telescopes are connected to the EVN Data Processor at JIVE at or near 1024 Mbps, and a wealth of ToO observations have been carried out outside of normal disc-recording sessions, showing the increased flexibility of the array using e-VLBI.
} 


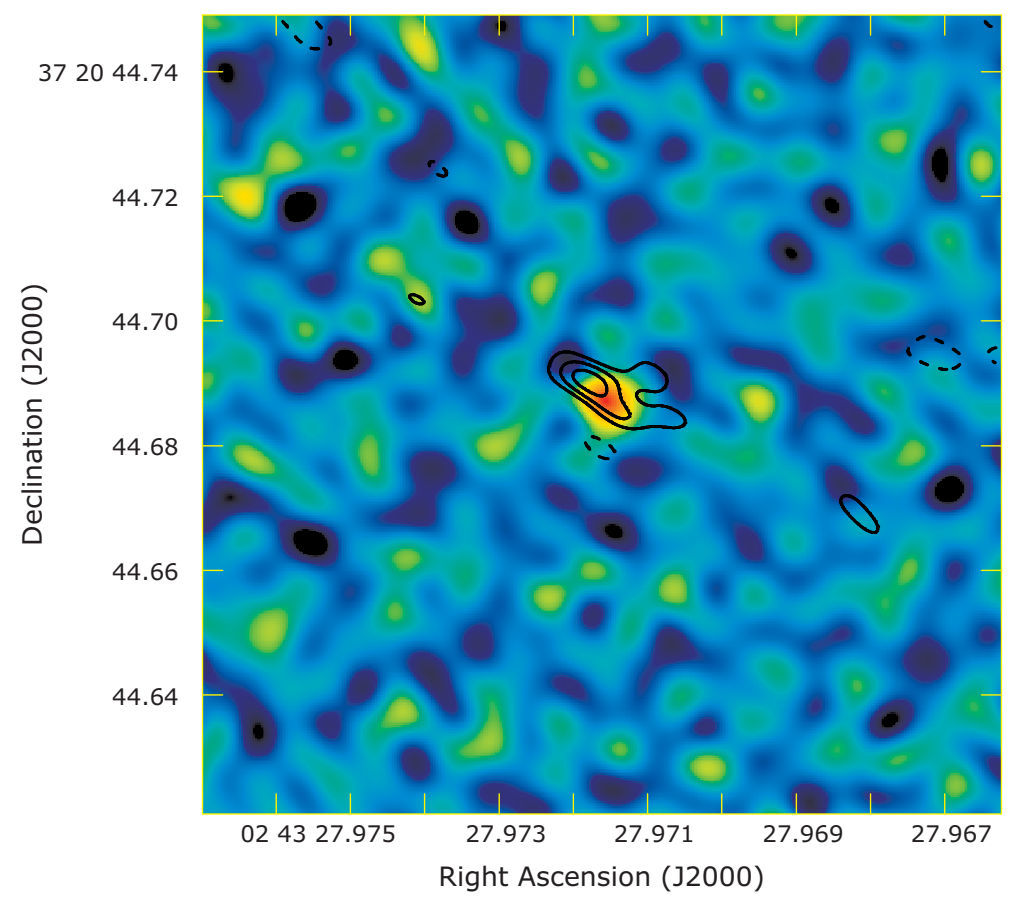

Figure 2: EVN (e-VLBI) and EVN+GBT observations of SN 2007gr. The colors, ranging from -214 to 441 microJy/beam, show the dirty map of SN $2007 \mathrm{gr}$ observed on 6-7 September 2007 at $5 \mathrm{GHz}$ with the 'e-EVN'. The off-source noise in the map is 75 microJy/beam, and the peak is 422 microJy/beam (5.6 $\sigma)$. The VLBI location of RA $=02 \mathrm{~h} 43 \mathrm{~m}$ 27s.97151, Dec $=+37 \mathrm{~d} 20^{\prime} 44^{\prime \prime} .6873$ (J2000) is consistent with the VLA coordinates RA=02h 43m 27s.972, Dec $=+37 \mathrm{~d} 20^{\prime} 44^{\prime \prime} .677$ (J2000) obtained at a lower resolution. The black contours show the naturally weighted and tapered EVN+GBT image of SN2007gr on 5-6 November 2007. At this epoch the off-source noise is 13 microJy/beam, and the peak is 60 microJy/beam $(4.7 \sigma)$. The image is centered at the position measured by the EVN at the first epoch. The restoring beam for this image is 15.26 x 6.85 mas in position angle of 53.3 degrees. Contours are drawn at -20, 20, 40, and 60 microJy/beam.

To further investigate the evolution of the ejecta on mas scales, we observed again on 5-6 November 2007 (age of about 85 days) with the EVN and the Green Bank Telescope (GBT). We followed exactly the same observing strategy as before, but the data in this case were recorded on disc at a rate of $1024 \mathrm{Mbps}(512 \mathrm{Mbps}$ at the GBT). The measured peak brightness of $60 \mu \mathrm{Jy} / \mathrm{beam}$ this time was significantly below the total flux density of $260 \mu \mathrm{Jy} / \mathrm{beam}$ measured by the WSRT, while they were fully consistent at the first epoch, within the errors. Radio maps obtained at the two epochs are shown in Figure 2.

\subsection{Background emission, phase errors, or expansion?}

To further support the WSRT measurements, we analysed archival VLA data, which were in agreement with our initial results (but see Soderberg et al. 2010). Our WSRT measurements are more sensitive, however, one needs to exclude the possibility that they may be contaminated by background emission in the galaxy due to the low resolution. We organized, therefore, late time WSRT observations of NGC 1058 (to be carried out shortly after the meeting) to constrain the 
amount of extended emission; these results will be addressed in a forthcoming paper.

Naturally, VLBI measurements are subject to phase coherence losses and calibration errors, which could explain the discrepancy between the peak brightness and the total flux density. This was carefully investigated in paper I and there the measured phase errors (using secondary calibrators) are much smaller than required to account for the difference. It appears that SN 2007gr must have been partially resolved on mas-scales. Taking the average of the major and minor axes of the beam, we derive a lower limit for the angular diameter size of 1.7 mas. This corresponds to a linear size of $2.7 \times 10^{17} \mathrm{~cm}$, and a lower limit of $>0.61 \mathrm{c}$ on the average apparent expansion speed.

\subsection{An otherwise normal supernova}

Apart from the mildly relativistic expansion of its radio emitting material, SN 2007gr appears normal, with photospheric expansion velocities smaller than those inferred in hypernovae (broadline type Ic supernovae), some of which are associated with GRBs. Energetically it is not peculiar either. Based on the spectral properties and the lower limit on the size we calculate the lower limit on the energy in synchrotron emitting material of $(0.7-1.3) \times 10^{46}(10 f)^{3 / 7}$ erg, where $f$ is the volume filling factor. The inferred non-relativistic transition time requires an isotropic equivalent kinetic energy of $\sim 10^{49} \mathrm{erg}$ for a reasonable external density. For a spherical flow the low radio luminosity would require a large deviation from equipartition (relativistic electrons and/or magnetic fields carrying very small fractions of the internal energy). Both the deviation from equipartition and the energy requirements are relaxed if the mildly relativistic ejecta are collimated into a narrow bipolar jet: $\sim 10^{47.5} \mathrm{erg}$ are required if the jet occupies a few percent of the total solid angle. Thus, our observations suggest that only a very small fraction $\left(\sim 10^{-4}\right)$ of the total explosion energy (of a few $10^{51} \mathrm{erg}$, estimated from optical observations) ends up in (mildly-) relativistic ejecta. Note that the minimum energy of about $10^{46}$ erg is just a few times smaller than observed in GRB 060218/SN 2006aj, but orders of magnitude lower than in hypernovae.

So far we have a very small sample but it is already clear that the population of strippedenvelope core-collapse supernovae is very diverse. It may very well be that SN2007gr is the typical case, with modest energy in moderately relativistic ejecta and no GRB, while a smaller fraction of type Ic supernovae have variable amounts of energy in relativistic ejecta and produce from very dim to powerful GRBs. In other words, the total energy of these explosions are similar $\left(\sim 10^{51}-10^{52} \mathrm{erg}\right.$ ), while the relativistic energy content varies dramatically (Kaneko et al. 2007). This would imply that in fact most of type Ic supernovae may produce bipolar jets, which might be tested with next generation radio instruments like the SKA.

\section{Conclusions}

We have shown VLBI observations of three type Ib/c supernovae. In the case of SN 2008D and SN 2007uy the expansion speed was at most mildly relativistic, while in SN 2007gr it appears at least midly relativistic. To constrain the speed of the ejecta better and detect more sources, we require orders of magnitude better signal-to-noise ratios. A large-configuration phase-II SKA with baselines up to about $3000 \mathrm{~km}$ will be suitable for this goal at frequencies between 5-10 GHz, although the resolution will be still too small in most of the cases to investigate the geometry of these supernovae. The quality of calibration will be much improved by simultaneous observations 
of nearby mJy-level sources, strong enough to serve as calibrators for the SKA. Additionally, the SKA with its wide field of view capability may potentially detect (and even discover, independently of optical observations) a large number of $\mathrm{SNe}$ and as a real-time and high resolution instrument will be an excellent instrument to monitor these sources, along with other types of extragalactic transients.

\section{Acknowledgements}

e-VLBI developments in Europe were supported by the EC DG-INFSO funded Communication Network Developments project 'EXPReS'. The European VLBI Network is a joint facility of European, Chinese, South African and other radio astronomy institutes funded by their national research councils. The WSRT is operated by ASTRON (Netherlands institute for Radio Astronomy) with support from the Netherlands Foundation for for Scientific Research (NWO). The National Radio Astronomy Observatory is operated by Associated Universities, Inc., under cooperative agreement with the National Science Foundation.

\section{References}

[1] E. Berger \& A. M. Soderberg, ATel 1353 (2008)

[2] M. F. Bietenholz, A. M. Soderberg, N. Bartel, Astrophys. J. 694, L6 (2009)

[3] B. E. Cobb, J. S. Bloom, D. A. Perley, Astrophys. J. 718, L150 (2010)

[4] R. M. Crockett, J. R. Maund, S. J. Smartt et al., Astrophys. J. 673, L155

[5] Y.-Z. Fan, B.-B. Zhang, D. Xu et al., arXiv 1004.5267 (2010)

[6] T. J. Galama, P. M. Vreeswijk, J. van Paradijs et al., Nature 395, 670 (1998)

[7] J. Granot, A. Loeb, Astrophys. J. 593, L81 (2003)

[8] J. Hjorth, J. Sollerman, P. Moeller et al., Nature 423, 847 (2003)

[9] Y. Kaneko, E. Ramirez-Ruiz, J. Granot et al., Astrophys. J. 654, 385 (2007)

[10] D. Madison, W. Li, CBET 1034, (2007)

[11] D. Malesani, G. Tagliaferri, G. Chincarini et al., Astrophys. J. 609, L5

[12] D. Malesani, J. Hjorth, P. Jakobsson et al., GCN 7169 (2008)

[13] P. A. Mazzali, S. Valenti, M. Della Valle et al., Science 321, 1185 (2008)

[14] P. Meszarosm, M. J. Rees, Astrophys. J. 482, L29 (1997)

[15] B. Paczinski, Acta Astronomica 51, 1 (2001)

[16] Z. Paragi, G. Taylor, C. Kouveliotou et al., Nature 463, 516 (2010)

[17] E. Pian, P. A. Mazzali, N. Masetti et al., Nature 442, 1011 (2006)

[18] L. S. Pilyugin, J. M. Vilchez, T. Contini, Astron. Astrophys. 425, 849 (2004)

[19] M. C. Shepherd, T. J. Pearson, G. B. Taylor, BAAS 26, 987 (1994)

[20] A. M. Soderberg, GCN 7178 (2008)

[21] A. M. Soderberg, E. Berger, K. L. Page et al., Nature 453, 469 (2008)

[22] A. M. Soderberg, A. Brunthaler, E. Nakar, R. A. Chevalier, M. F. Bietenholz, arXiv 1005.1932 (2010) 
[23] K. Z. Stanek et al., Astrophys. J. 591, L17 (2003)

[24] R. L. C. Starling, K. Wiersema, A. J. Levan et al., arXiv 1004.2919 (2010)

[25] A. Szomoru, Proceedings of Science, (IX EVN Symposium)040 (2008)

[26] C. C. Thoene, D. Malesani, J. Hjorth et al., GCN 7161 (2008)

[27] S. Valenti, D. Fugazza, E. Maiorano et al., GCN 7171 (2008)

[28] S. Valenti, N. Elias-Rosa, S. Taubenberger et al., Astrophys. J. 637, 155 (2008)

[29] A. J. van der Horst, C. Kouveliotou, R. .A. M. .J. Wijers \& A. Kamble, GCN 7190 (2008)

[30] R. A. M. J. Wijers, M. J. Rees, P. Meszaros, Mon. Not. Roy. Astron. Soc. 288, L51

[31] S. E. Woosley, Astrophys. J. 405, 273 (1993) 